\title{
Determination of Buprofezin Residues in Rice and Fruits Using HPLC with LC/MS Confirmation
}

\author{
Young Deuk Lee ${ }^{*}$ and Sang Won Jang \\ Division of Life and Environmental Science, Daegu University, Gyeongbuk 712-714, Korea \\ (Received September 14, 2010, Accepted September 24, 2010)
}

\begin{abstract}
A high-performance liquid chromatographic (HPLC) method was developed to determine buprofezin residues in hulled rice and fruits. The buprofezin residue was extracted with acetone and the extract was stepwise purified by liquid-liquid partition and Florisil column chromatography. For rice samples, acetonitrile/ $n$-hexane partition was additionally employed to remove nonpolar lipids. Reversed phase HPLC using an octadecylsilyl column was successfully applied to separate buprofezin from sample co-extractives, as detected by ultraviolet absorption at $250 \mathrm{~nm}$. Recovery experiment at the limit of quantitation validated that the proposed method could evidently determine the buprofezin residue at the level of $0.02 \mathrm{mg} / \mathrm{kg}$. Mean recoveries from hulled rice, apple, pear, and persimmon samples fortified at three tenfold levels were in the range of 80.8 85.2\%, 89.1 98.4\%, 88.8 95.7\% and 90.8 96.2\%, respectively. Relative standard deviations of the analytical method were all less than $5 \%$, irrespective of sample types. A selected-ion monitoring LC/mass spectrometry with positive electrospray ionization was also provided to sensitively confirm the suspected residue.
\end{abstract}

Key Words: Analysis, Buprofezin, Fruit, Residue, Rice

\section{INTRODUCTION}

Buprofezin [(Z)-2-tert-butylimino-3-isopropyl-5-phenyl1,3,5-thiadiazinan-4-one] is a persistent insecticide and acaricide with larvicidal action by inhibiting chitin biosynthesis and effects on the hormone levels of nymphs (Tomlin, 2009). As predicted by its mode of action, the slow emergence of efficacy leads buprofezin to be mostly involved as a component of combined insecticides. Diverse combined insecticides containing buprofezin are currently available for dilute spray on rice, fruits and vegetables to control various insects and mites (KCPA, 2010). Buprofezin is known to be contact and stomach poison which is not translocated in the plant. Hence, the potential hazard resulted from its residue level could be entirely evaluated by

*연락저자:

Tel: +82-53-850-6753 Fax: +82-53-850-6759

E-mail: ydpechem@daegu.ac.kr persistence of buprofezin applied in situ rather than its physical movement in the nearby environment. Taken in conjunction with the persistence in rice and fruits, use of buprofezin inevitably results in potential occurrence of its terminal residues in the harvest. As rice and fruits are consumed in large quantity, particularly in raw state in case of fruits, residue evaluation of buprofezin is of considerable importance to ensure safety of the harvest. Therefore, development of a highly reliable method is critical for estimation of the residue in rice and fruits.

As maximum residue limits (MRLs) of buprofezin

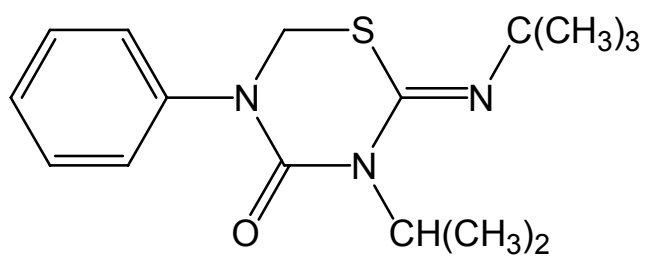

Fig. 1. Molecular structure of buprofezin. 
in foods range $0.05 \sim 1.0 \mathrm{mg} / \mathrm{kg}$ (KFDA, 2009), highly sensitive method is required to meet $0.02 \mathrm{mg} / \mathrm{kg}$ of limit of quantitation (LOQ) or less to fulfill the Guideline on Method of Pesticide Residue Analysis (CAC, 2003; RDA, 2004). Owing to the strict regulation of MRLs, certainty of analytical methodology should be also lied on providing unambiguous evidence to confirm both the identity and the quantity of any detected residue for the official decision on MRL violation. Buprofezin is not yet included as an analyte in KFDA and US FDA official multi-residue analytical method in foods (KFDA, 2009; US FDA, 1999). Recently, several multi-residue methods, for the purpose of screening and monitoring of diverse pesticide residues which included buprofezin have been reported using gas-liquid chromatography (GLC)/ nitrogen-phosphorus detection (NPD)/mass spectrometry (MS) (Borges et al., 2009; Santos et al., 2008) or liquid chromatography (LC)/MS (Soler et al., 2005; Lacina et. al., 2010). However, because buprofezin showed low sensitivity in GC/MS (more than 0.1 $\mathrm{mg} / \mathrm{kg}$ of LOQ) and poor repeatability in LC/MS (9-19\% of analytical error), those methods could not be employed as official tools for precise quantitation. A few of individual methods had been published using GLC/NPD (PRARG, 2006; KFDA 2009). However, employment of NPD is not recommended for precise quantitation of pesticide residues due to poor stability of the detector response (US FDA, 1999). Even though KFDA individual method is available using GLC with NPD, LOQ of $0.05 \mathrm{mg} / \mathrm{kg}$ is unsatisfactory to cover lowest MRLs.

The present paper describes a new individual method for buprofezin residues in rice and fruit samples using high-performance liquid chromatography, preferably for the official routine analysis. The method was developed not only to provide reliability higher than current methods but to achieve requisite sensitivity and readiness for analytical operation.

\section{MATERIALS AND METHODS}

\section{Chemicals}

Analytical standard of buprofezin (99.7\% pure) was kindly supplied by Nihon Nohyaku Co., Japan. Stock standard solution of $500 \mathrm{mg} / \mathrm{L}$ was prepared in acetonitrile. The stock solution was stable at $4^{\circ} \mathrm{C}$ for at least 6 months. Working solutions for fortification and HPLC calibration were freshly prepared in acetonitrile and acetonitrile/water mixture (70/30, $\mathrm{v} / \mathrm{v})$, respectively, whenever necessary. Florisil (60 100 mesh, pesticide residue grade) was purchased from J.T. Baker (USA) and activated at $130^{\circ} \mathrm{C}$ for more than $5 \mathrm{~h}$ prior to use. Acetonitrile and deionized water were HPLC grade. All other solvents were pesticide residue grade or reagent grade freshly redistilled in glass. All other reagents were reagent grade unless specified.

\section{Rice and fruit samples}

Hulled rice of Chuchung variety from an organically-grown field where no pesticides had been applied was purchased in a commercial market. At maturity control fruit samples were collected in bulk from orchard fields in Gyeongbuk Province, Korea, where no buprofezin had been applied. Varieties and orchard locations were as follows: apple, Fuji from Chilgok area; pear, Singo from Youngchun area; persimmon, Buyou from Chilgok area. Composite rice and fruit samples were prepared in compliance with the instructions in Korean Test Guidelines for Pesticide Persistence (RDA, 2004). Rice grains were finely pulverized to pass 40-mesh sieve using a Wiley-type mill. Apple, pear and persimmon fruits were minced after removing and discarding the hilum and ovary portions. Each analytical sample was stored frozen at $-20^{\circ} \mathrm{C}$ until analysis.

\section{Extraction and partition}

To a $25 \mathrm{~g}$ of rice sample in a $500-\mathrm{mL}$ homogenizer cup was added $20 \mathrm{~mL}$ of distilled water to moisten the sample. After brief shaking and standing for 30 min, $100 \mathrm{~mL}$ of acetone was added. A 25-g portion of fruit sample was weighed in the homogenizer cup, and $100 \mathrm{~mL}$ of acetone was added. The mixture was then macerated at $10,000 \mathrm{rpm}$ for $2 \mathrm{~min}$ in a high-speed homogenizer (Nihonseiki Kaisha AM-8, Japan). The homogenate was suction-filtered through the filter paper (Toyo No. 6, Japan) on porcelain Büchner funnel. The cup and filter cake were washed with fresh $50 \mathrm{~mL}$ of acetone, and the rinsate was combined with the previous filtrate. The filtrate was quantitatively transferred to a 1-L separatory funnel, 
and sequential addition of $100 \mathrm{~mL}$ of $n$-hexane, 50 $\mathrm{mL}$ of saturated sodium chloride solution, and 450 $\mathrm{mL}$ of distilled water was followed. After vigorous shaking for $1 \mathrm{~min}$ and standing until two layers clearly separated, the lower aqueous phase was discarded. The hexane phase was dried over $20 \mathrm{~g}$ of anhydrous sodium sulfate layer, collected in a 250-mL distilling flask, and evaporated just to dryness in vacuo at $40^{\circ} \mathrm{C}$. The residue was dissolved in $10 \mathrm{~mL}$ of $n$-hexane and subjected to Florisil column chromatography. For rice sample, the residue was dissolved in $40 \mathrm{~mL}$ of $n$-hexane saturated with acetonitrile and transferred to a $250-\mathrm{mL}$ separatory funnel. The hexane phase was then extracted twice with $30 \mathrm{~mL}$ portions of acetonitrile saturated with $n$-hexane. The acetonitrile extract was combined in a 125-mL distilling flask, and evaporated just to dryness in vacuo at $40^{\circ} \mathrm{C}$. The residue was dissolved in $10 \mathrm{~mL}$ of $n$-hexane and subjected to Florisil column chromatography.

\section{Florisil column chromatography}

A chromatographic column $(11 \mathrm{~mm}$ i.d. $\times 40 \mathrm{~cm})$ was plugged with glass wool, dry packed with $5 \mathrm{~g}$ of activated Florisil, and topped with ca. $2 \mathrm{~cm}$ layer of anhydrous sodium sulfate. The column was pre-washed by passing $25 \mathrm{~mL}$ of $n$-hexane until the solvent level reached the top of the sodium sulfate layer. A $5 \mathrm{~mL}$ aliquot of the hexane extract from the partition step was poured into the column and the column wall was rinsed twice with $2 \mathrm{~mL}$ portions of $n$-hexane. When the liquid drained to sodium sulfate layer, the column was eluted with $50 \mathrm{~mL}$ of dichloromethane/ acetonitrile/n-hexane mixture $(50 / 0.5 / 49.5, \mathrm{v} / \mathrm{v} / \mathrm{v})$, and the fraction was discarded. The column was then eluted with $50 \mathrm{~mL}$ of dichloromethane/acetonitrile/nhexane mixture $(50 / 1.5 / 48.5, \mathrm{v} / \mathrm{v} / \mathrm{v})$. The eluate was collected, rotary-evaporated just to dryness at $40^{\circ} \mathrm{C}$, and the residue was reconstituted with $5 \mathrm{~mL}$ of acetonitrile/water mixture $(70 / 30, \mathrm{v} / \mathrm{v})$ for HPLC determination.

\section{High Performance liquid chromatography}

High-performance liquid chromatography (HPLC) was performed using a HPLC system consisted of Waters (USA) 515 pumps, 680 gradient controller, 2489 UV/VIS absorbance detector, Agilent (USA)
1100 autosampler and column oven, and Younglin (Korea) Autochro $2000^{\circledR}$ data module/processing software. Hypersil Gold ${ }^{\circledR}$ C18 (4.0 mm i. d. $\times 250$ $\mathrm{mm}, 5 \mu \mathrm{m}$ spherical, Thermo, USA) was used as the analytical column. Operating parameters used for the determination of buprofezin residues were as follows; column temperature $40^{\circ} \mathrm{C}$; mobile phase, acetonitrile/ water $(70 / 30, \mathrm{v} / \mathrm{v})$, isocratic; flow rate, $1.0 \mathrm{~mL} / \mathrm{min}$; detection, UV absorption at $250 \mathrm{~nm}, 0.008$ AUFS; sample size, $20 \mu \mathrm{L}$. Under these conditions, retention time of buprofezin was $9.1 \mathrm{~min}$.

\section{Validation of the analytical method}

Recovery experiments were run on control samples to validate the analytical method proposed for buprofezin residues. Prior to extraction, series of control samples were fortified with buprofezin standard solution in acetonitrile at specified concentrations. After standing for $2 \mathrm{~h}$, analytical procedures mentioned above were carried out to produce quality assurance data.

\section{Confirmation of the residue}

LC/MS was used to confirm the buprofezin residue in crop extracts. Buprofezin was also separated from sample co-extractives on C18 columns containing the packing material identical to HPLC determination. Operating parameters of LC/MS were carefully optimized for maximum ionization of buprofezin as indicated in Table 1.

\section{RESULTS AND DISCUSSION}

Taking a glance at physicochemical properties of buprofezin, which show high lipophilicity of $\log \mathrm{P}_{\mathrm{ow}}$ (n-octanol-water partition coefficient) 4.9 and vapor pressure of $4.2 \times 10^{-2} \mathrm{mPa}\left(20^{\circ} \mathrm{C}\right)$ (Tomlin, 2009), GLC with NPD or flame-photometric detector (FPD) seems to be preferable to analyze the residues at MRL level. However, thiadiazine ring and imino group in the molecule may cause adsorption or heat-lability which leads to peak asymmetry and poor reproducibility (Lee and Lee, 1998; Lee and Kwon, 2004). As vapor pressure of buprofezin is relative low, high column temperature is quite necessary to elute the compound for the practical time of analysis. In this case, the undesirable effect would be more severe. Furthermore, consistent quantitation may not be guaranteed because 
Table 1. LC/ MS operating parameters for the confirmation of buprofezin residues

\begin{tabular}{lll}
\hline \multicolumn{1}{c}{ Instrument } & \multicolumn{2}{c}{ Thermo Finnigan (USA) Surveyor MSQ Plus LC/MS } \\
\hline Mode & \multicolumn{1}{c}{ ESI positive-ion mode } & \multicolumn{1}{c}{ APCI positive-ion mode } \\
\hline Column & Hypersil Gold ${ }^{\circledR} \mathrm{C} 18$ & Hypersil Gold ${ }^{\circledR} \mathrm{C} 18$, \\
& $2.1 \mathrm{~mm}$ i.d. $\times 150 \mathrm{~mm}, 3 \mu \mathrm{m}$ & $4.0 \mathrm{~mm}$ i.d. $\times 250 \mathrm{~mm}, 5 \mathrm{~m}$ \\
Mobile phase & $\mathrm{CH}_{3} \mathrm{CN} /$ water $(75 / 25)$ & $\mathrm{CH}_{3} \mathrm{CN} /$ water $(80 / 20)$ \\
Flow rate & $0.2 \mathrm{~mL} / \mathrm{min}$ & $1.0 \mathrm{~mL} / \mathrm{min}$ \\
Column temp. & $40^{\circ} \mathrm{C}$ & $40^{\circ} \mathrm{C}$ \\
Sample size & $5 \mu \mathrm{L}$ & $10 \mu \mathrm{L}$ \\
Cone voltage & $45 \mathrm{~V}$ & $50 \mathrm{~V}$ \\
Needle voltage & $3.0 \mathrm{kV}$ & - \\
Corona current & - & $3.0 \mu \mathrm{A}$ \\
Probe temp. & $500^{\circ} \mathrm{C}$ & $600^{\circ} \mathrm{C}$ \\
$\mathrm{N}_{2}$ gas pressure & $75 \mathrm{psi}$ & $45 \mathrm{psi}$ \\
Mass range $(\mathrm{m} / \mathrm{z})$ & $200 \sim 400$ & $200 \sim 400$ \\
SIM ion $(\mathrm{m} / \mathrm{z})$ & 306 & 306 \\
SIM span $(\mathrm{m} / \mathrm{z})$ & 0.5 & 0.5 \\
\hline
\end{tabular}

the stability of NPD is known to be just fair and large fluctuation of response is frequently found being entirely dependent on alkaline bead condition (US FDA, 1999). FPD in sulfur mode is presumed to be insufficient sensitivity as buprofezin possesses only one sulfur atom. Considering these drawbacks, GLC is inadequate for the precise determination of buprofezin residues for the official purpose. A study was therefore tried to develop an alternative method using HPLC to ensure high reproducibility along with enhanced sensitivity.

When reversed-phase HPLC on an octadecylsilyl column was applied, buprofezin showed a sharp symmetrical peak under the mobile phase of acetonitrile/ water mixture. In the range of $50 \%$ to $80 \%$ of acetonitrile contents in water, its capacity factor increased almost double as acetonitrile contents decreased by $10 \%$, while the peak shape was kept totally symmetrical. This indicated that buprofezin exists as neutral form in the mobile phase of acetonitrile/water mixture and thus there was no need for ion-suppression (Snyder et al., 1997).

As buprofezin was not readily oxidized nor reduced and had no fluorophore, ultraviolet absorption detector was the only choice among common HPLC detectors. Buprofezin standard solution $(10 \mathrm{mg} / \mathrm{L})$ in acetonitrile or $50 \%$ aqueous acetonitrile was scanned in the range of 210 400 nm using Hewlett Packard (USA) Model

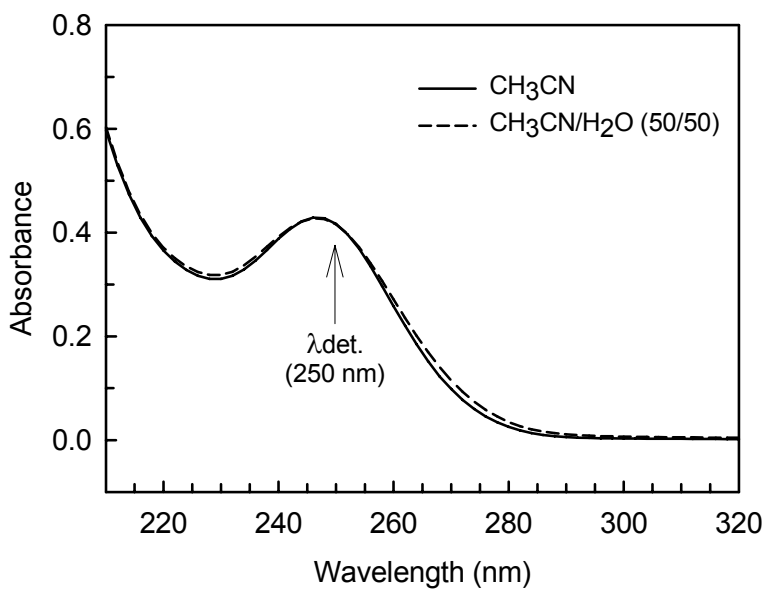

Fig. 2. UV absorption spectra of buprofezin.

8452A photodiode-array UV/VIS spectrophotometer. As shown in Fig. 2, $\lambda$ max of buprofezin was found at $246 \mathrm{~nm}$ and molar absorptivity at $\lambda \max$ was $1.31 \times$ $10^{4} \mathrm{~cm}^{-1} \mathrm{M}^{-1}$. No spectral change between two solvents was observed. This also confirmed no dissociation of buprofezin in the HPLC mobile phase of acetonitrile/ water mixture. To enhance specificity, wavelength of determination was setup to be as long as possible with minimal loss of sensitivity (Lee and Kwon, 1999). Absorptivity at determination wavelength of $250 \mathrm{~nm}$ was $1.27 \times 10^{4} \mathrm{~cm}^{-1} \mathrm{M}^{-1}$ equivalent to $97 \%$ of absorptivity at $\lambda \max$.

Under optimized HPLC parameters, LOQ of bupro- 
fezin was $1.0 \mathrm{ng}$ at signal to noise ratio $(\mathrm{S} / \mathrm{N}) \geq 10$. Although molar absorptivity of buprofezin was high enough for requisite sensitivity and determination was performed at longer wavelength for specificity, there are always possibilities of interference caused by sample co-extractives, particularly in residue analysis of diverse type of samples. The study was, therefore, mainly focused on the development of efficient but simple cleanup methods.

Based on the nonpolar and undissociated nature of buprofezin, acetone was used to extract the residue from food samples, as its extraction efficiency for nonpolar neutral compounds had been validated in many official methods (US FDA, 1999; Zweig and Sherma, 1986). The acetone extract was then diluted with large amount of saline and the aqueous phase was directly partitioned with $n$-hexane to recover the analyte. This partition procedure is quite simple to operate with advantage of omitting tedious concentration step of the extract. Single hexane partition was sufficient to recover more than $99 \%$ of buprofezin. No buprofezin was found at all in the repeated partition with hexane.

For rice sample, an attempt was done at partition step to remove nonpolar co-extractives, mainly lipids, employing modified method of acetonitrile $/ n$-hexane partition (US FDA, 1999; Zweig and Sherma, 1986). In the hexane partition of the acetone extract, dried amount of the rice extract partitioned to the hexane phase was more than $200 \mathrm{mg} / 25 \mathrm{~g}$ sample, while that of fruit samples were all less than $50 \mathrm{mg}$ as indicated in Table 2. It was expected that excess co-extractives from the rice sample would evidently cause overloading of Florisil column chromatography as well as severe interference in instrumental determination (Lee and Lee, 1998; Lee and Kwon, 2004). When acetonitrile/ $n$-hexane partition was tried, 92 and $98 \%$ of buprofezin was recovered by double and triple acetonitrile partitions, respectively. On the other hand, removal rate of co-extractives which remained in the hexane phase was 67 and 56\% at corresponding number of partition. To provide rigorous purification of the extract within acceptable loss of buprofezin, double partition with acetonitrile was adopted.

Adsorption chromatography was applied to further purify the extracts. As buprofezin is a nonpolar neutral compound, Florisil and dichloromethane/ acetonitrile/ n-hexane mixtures were used as adsorbent and elution solvents, respectively (US FDA, 1999). As shown in Table 3, elution pattern of buprofezin on Florisil

Table 2. Effect of acetonitrile/ $n$-hexane partition (AHP) on the removal of nonpolar co-extractives from the rice extract

\begin{tabular}{ccccc}
\hline \multirow{2}{*}{$\begin{array}{c}\text { Partition frequency } \\
\text { with } \mathrm{CH}_{3} \mathrm{CN}\end{array}$} & $\begin{array}{c}\text { Buprofezin } \\
\text { recovery (\%) }\end{array}$ & Before AHP & After AHP & Removal (\%) \\
\hline 2 & 92 & 223 & 74 & 67 \\
3 & 98 & 210 & 92 & 56 \\
\hline
\end{tabular}

${ }^{1}$ Mean of duplication.

${ }^{2}$ After hexane partition of the acetone extract diluted with sali

Table 3. Elution profile of buprofezin on Florisil column ${ }^{1}$

\begin{tabular}{ccccc}
\hline \multirow{2}{*}{ Elution solvent $^{2}$} & \multicolumn{5}{c}{ Buprofezin recovery (\%) } \\
\cline { 2 - 5 } & $0 \sim 25 \mathrm{~mL}$ & $25 \sim 50 \mathrm{~mL}$ & $50 \sim 75 \mathrm{~mL}$ & Total \\
\hline $50 / 0.5 / 49.5$ & $\mathrm{NE}^{4}$ & $\mathrm{NE}$ & 1 & 1 \\
$50 / 1.5 / 48.5$ & 11 & 68 & 13 & 92 \\
$50 / 2.0 / 48.0$ & 49 & 39 & 7 & 95 \\
$50 / 1.5 / 48.5^{3}$ & 55 & 42 & 2 & 99 \\
\hline
\end{tabular}

${ }^{1}$ Activated Florisil, $5 \mathrm{~g}$ was pre-washed with $25 \mathrm{~mL}$ of $\mathrm{n}$-hexane.

${ }^{2}$ Dichloromethane/acetonitrile/n-hexane mixture (v/v/v).

${ }^{3}$ Pre-eluted with $50 \mathrm{~mL}$ of dichloromethane/acetonitrile/n-hexane (50/0.5/49.5), discarded and then eluted with $75 \mathrm{~mL}$ of dichloromethane/acetonitrile/n-hexane (50/1.5/48.5).

${ }^{4}$ Not eluted. 
column was investigated at different solvent strength. While no loss of analyte was ensured, gradient elution scheme consisted of serial washing and collecting fractions was optimized to sharply separate buprofezin from sample co-extractives.

Coupling with the proposed partition and adsorption chromatography, rice and fruit extracts appeared so well purified that nearly colorless solutions were provided for final HPLC determination. Typical HPLC chromatograms of rice and fruit extracts are shown in Fig. 3.

All the HPLC chromatograms of extracts from control samples showed free of interference near buprofezin elution. Co-extractive peaks eluted later than buprofezin were negligible to enable consecutive HPLC determination at $15 \mathrm{~min}$ interval. In consequence of recovery experiment, LOQ of the proposed method was apparently verified to be $0.02 \mathrm{mg} / \mathrm{kg}$ for both of rice and fruit samples based on $3 \%$ full scale deflection ( $S / N \geq 10$ ). These sensitivities were high enough to quantitate up to $1 / 10 \sim 1 / 20$ of MRLs ( 0 . 5 1 $\mathrm{mg} / \mathrm{kg}$ ) established for buprofezin in rice and fruits (KFDA, 2009).

Percent recoveries of buprofezin generated during the validation of analytical methods are presented in Table 4. Overall recoveries performed at three
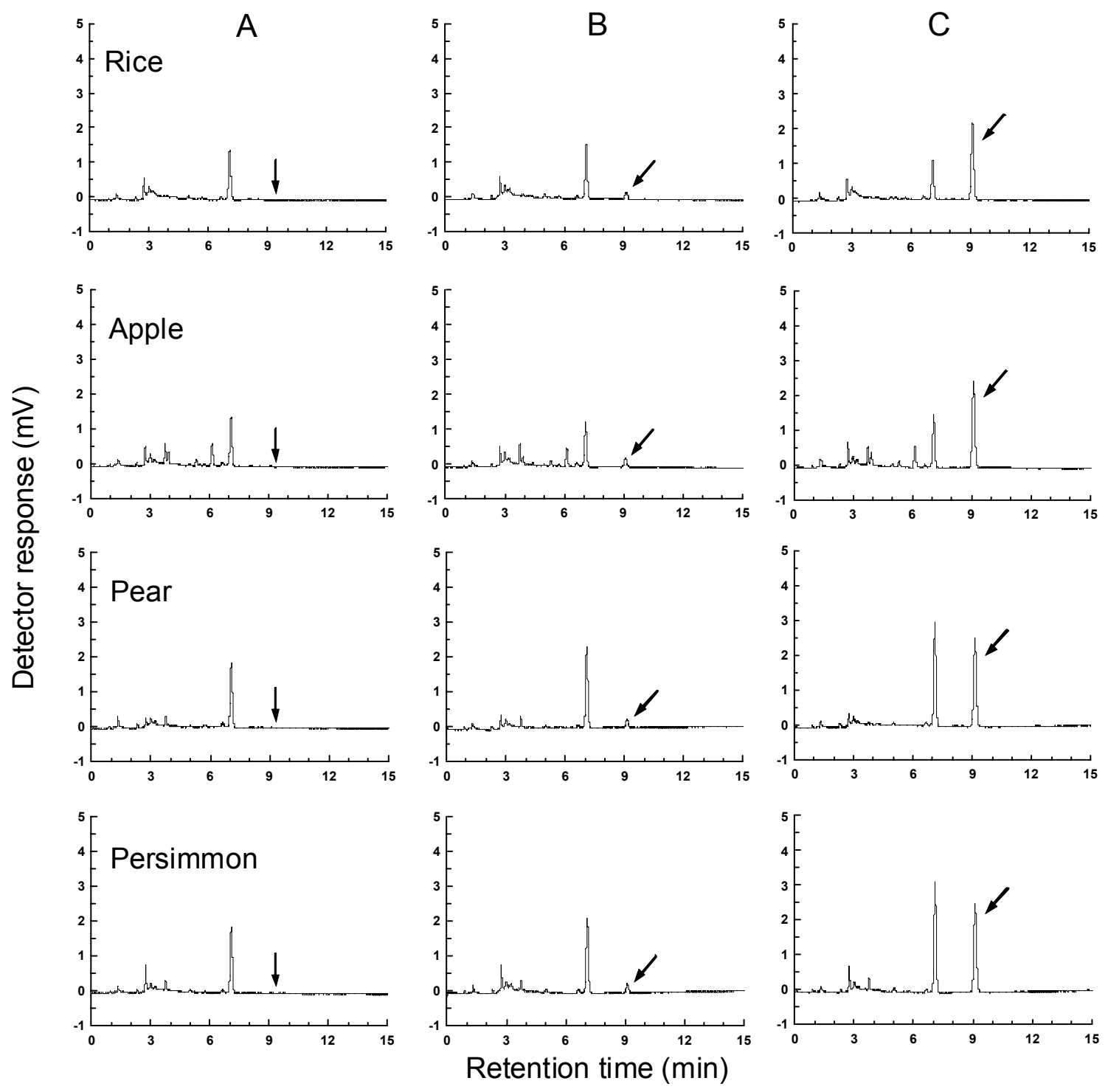

Fig. 3. HPLC chromatograms of crop extracts for the analysis of buprofezin residues. A, control; B, fortified at $0.02 \mathrm{mg} / \mathrm{kg}$ (LOQ); C, fortified at $0.2 \mathrm{mg} / \mathrm{kg}$. 
Table 4. Recoveries and LOQs of the analytical method

\begin{tabular}{|c|c|c|c|}
\hline Crop & Fortification $(\mathrm{mg} / \mathrm{kg})$ & Recovery \pm SD $(\%)^{1}$ & LOQ $(\mathrm{mg} / \mathrm{kg})^{2}$ \\
\hline \multirow[t]{3}{*}{ Hulled rice } & 0.02 & $80.8 \pm 1.7$ & 0.02 \\
\hline & 0.2 & $82.1 \pm 1.2$ & \\
\hline & 2.0 & $85.2 \pm 1.2$ & \\
\hline \multirow[t]{3}{*}{ Apple } & 0.02 & $98.4 \pm 1.8$ & 0.02 \\
\hline & 0.2 & $89.1 \pm 1.2$ & \\
\hline & 2.0 & $90.5 \pm 1.0$ & \\
\hline \multirow[t]{3}{*}{ Pear } & 0.02 & $88.8 \pm 3.3$ & 0.02 \\
\hline & 0.2 & $95.7 \pm 0.5$ & \\
\hline & 2.0 & $95.2 \pm 1.5$ & \\
\hline \multirow[t]{3}{*}{ Persimmon } & 0.02 & $90.0 \pm 3.0$ & 0.02 \\
\hline & 0.2 & $96.2 \pm 0.9$ & \\
\hline & 2.0 & $95.1 \pm 1.2$ & \\
\hline
\end{tabular}

${ }^{1}$ Mean values of triplicate samples with standard deviations.

${ }^{2} \mathrm{LOQ}$ at $3 \%$ FSD $(\mathrm{S} / \mathrm{N} \geq 10)$.

fortification levels in triplicate averaged $82.7 \pm 2.3 \%$ $(n=9), 92.6 \pm 4.5 \%(n=9), 93.2 \pm 3.8 \%(n=9)$ and $93.8 \pm 3.3 \%$ $(n=9)$ for hulled rice, apple, pear and persimmon samples, respectively. Sightly lower recoveries were observed in rice samples due to some loss of the analyte during acetonitrile/ $n$-hexane partition. Relative standard deviations (RSD) of recoveries were all less than $5 \%$, irrespective of sample types and spiking levels, indicating that the method could be reproducibly applied to analyze buprofezin residues in rice and fruit samples.

Confirmatory tests play an essential part in the analysis of pesticide residues. At present, there are few analytical techniques available that alone can unequivocally identify pesticide residues in trace quantities. Even though the proposed method was carefully designed to remove and separate every interference as much as possible, there is still high probability of the occurrence of other pesticides in the extract with very similar retention times. Unexpected interference could be also encountered with co-extractives of different sample origin and artifacts from laboratory contamination. The purpose of a confirmatory test is to verify the result of an initial analysis and is usually carried out under different experimental conditions, mainly in the form of different measuring devices (Das, 1981; UD FDA 1999). Hence, in the present study, the confirmatory test using LC/MS was additionally developed to validate the identity of the detected buprofezin.

Buprofezin was readily ionized to form protonated molecular ion, $(\mathrm{M}+\mathrm{H})^{+}$, under positive-ion modes of electrospray ionization (ESI) and atmospheric-pressure chemical ionization (APCI), presumably resulted from lone pair in nitrogen as proton acceptor (Ardrey, 2003). On the condition that each ionization parameter was optimized, total-ion chromatograms were obtained to have similar retention time using columns of packing material same to HPLC determination (Fig. 4).

Mass spectra of buprofezin under ESI and APCI (Fig. 5) appeared identical each other and single base peak representing $(\mathrm{M}+\mathrm{H})^{+}$ion was found almost except small fragment ion, $\left(\mathrm{M}-\mathrm{C}_{6} \mathrm{H}_{5} \mathrm{NCH}_{2}\right)^{+}$, at $\mathrm{m} / \mathrm{z}=201$. Isotope abundance of the ion was also coincident to the expected value from chemical formula of the molecule (McLafferty and Turecek, 1993).

Buprofezin exhibited 30 times higher sensitivity in $\mathrm{ESI}(+)$ mode than that in $\mathrm{APCI}(+)$. Therefore selected-ion monitoring (SIM) was conducted under $\mathrm{ESI}(+)$ mode based on $(\mathrm{M}+\mathrm{H})^{+}$ion at $m / z=306$. As 


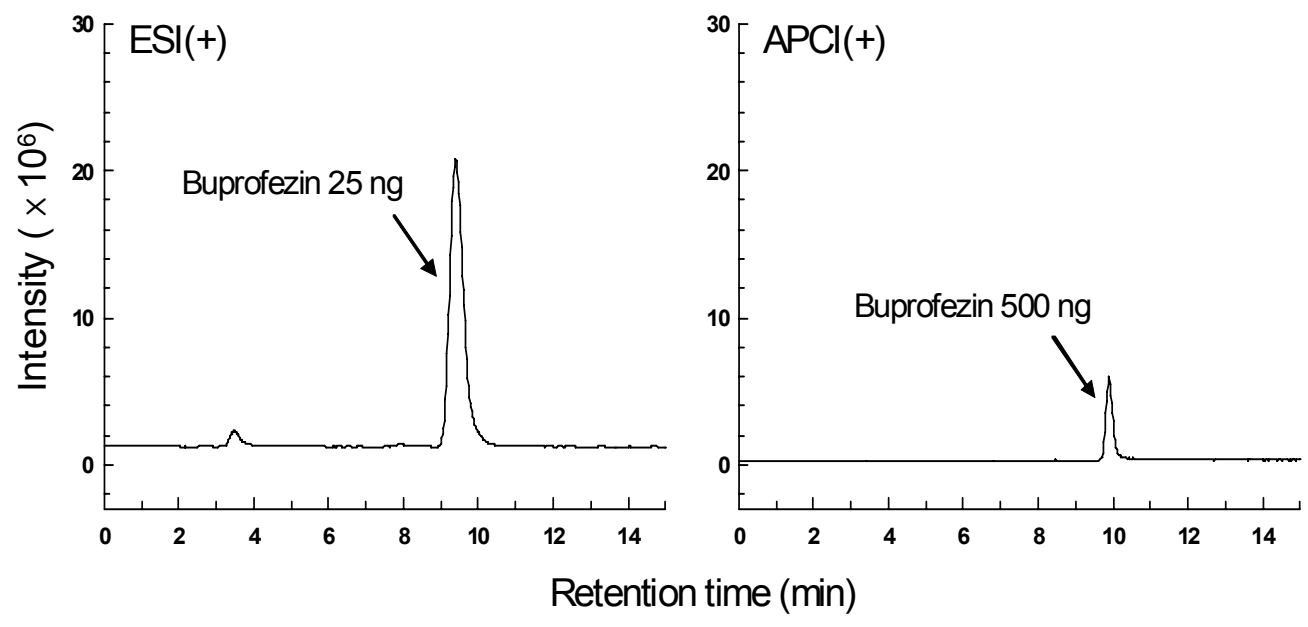

Fig. 4. LC/ MS total-ion chromatograms of buprofezin.

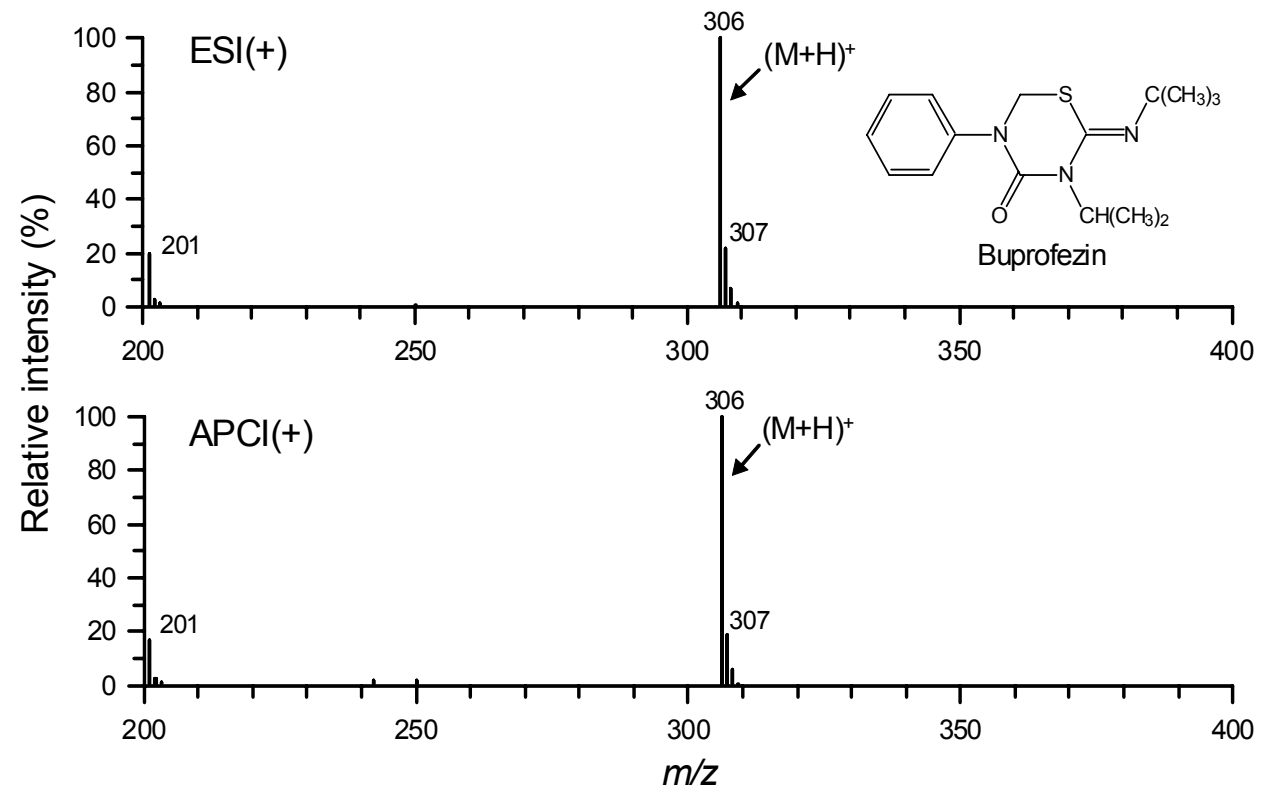

Fig. 5. Mass spectra of buprofezin.

shown in Fig. 6, identity of buprofezin at LOQ level was evidently confirmed with no interference. LC/MS is at least 10-fold more sensitive than HPLC/UVD and could also be used an alternative instrument for detection of buprofezin residues. Ion-suppression by the co-extractives was not so serious that quantitation would be also possible if matrix-matched calibration was followed.

The proposed method satisfies criteria of the analytical method for pesticide residues, which are 7 $0 \sim 120 \%$ of recovery range, less than $10 \%$ of RSD, and LOQs more sensitive than $0.05 \mathrm{mg} / \mathrm{kg}$ along with half of lowest MRLs, on Test Guidelines for Pesticide Residue Analysis notified by Rural Development Administration (RDA, 2004) as well as recommended by Codex Alimentarius Commission (CAC, 2003). Analytical procedures do not require any special apparatus or instruments but consist of currently available techniques familiar to the residue analyst as well. The method is so rapid to operate that one experienced person can analyze 12 samples per day. Therefore, authors suggest that the proposed method could be routinely applied to the official analysis of buprofezin residues in rice and fruit samples. 
A
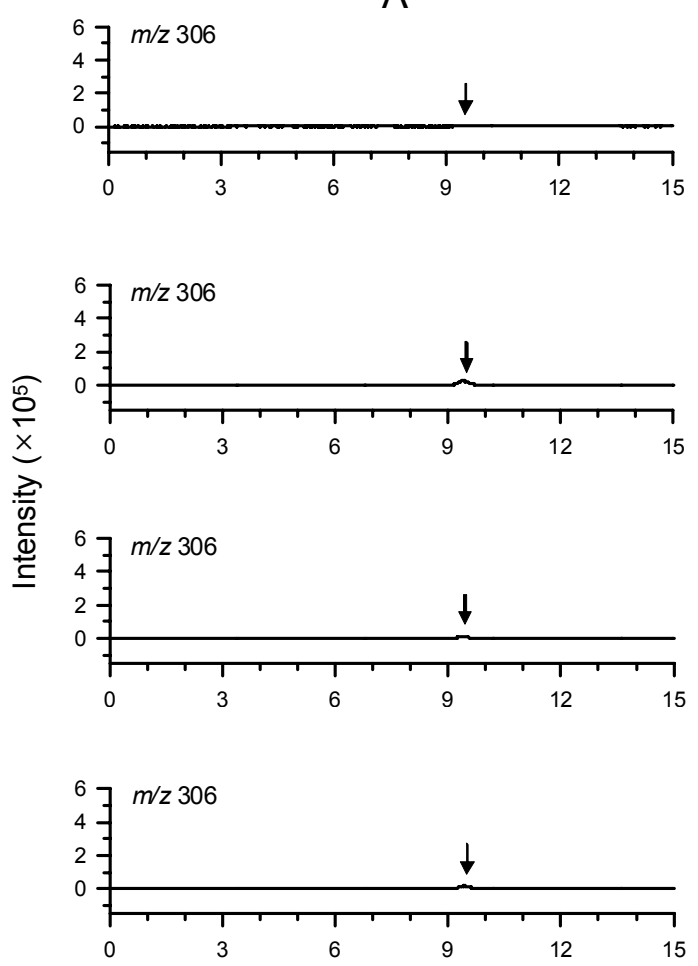

B
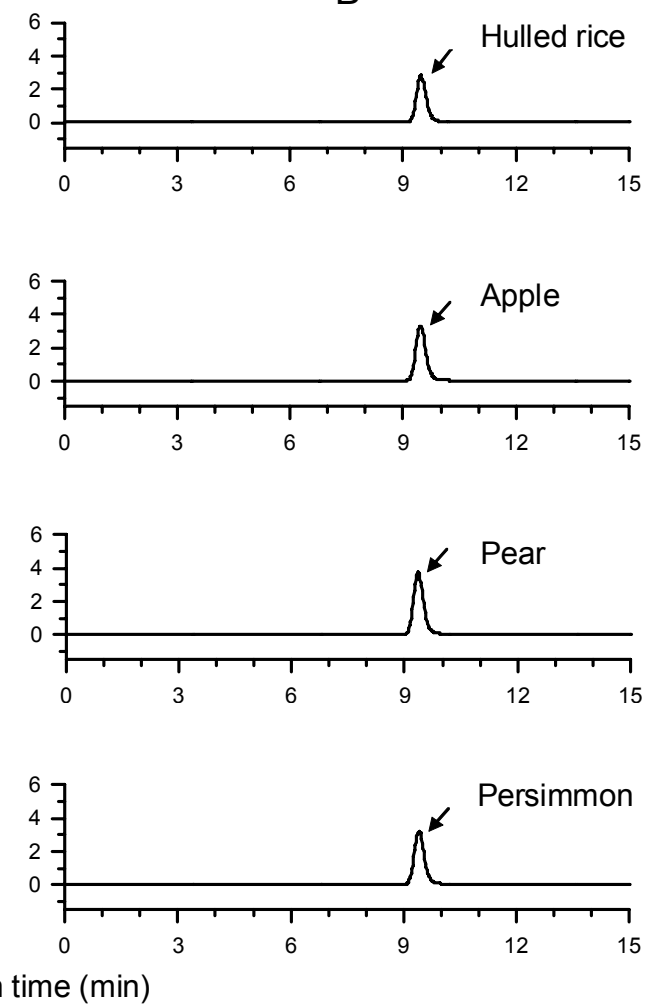

Fig. 6. LC/ MS ESI(+) SIM chromatograms of sample extracts for confirmation of buprofezin residues. A, control; B, fortified with buprofezin at LOQ $(0.02 \mathrm{mg} / \mathrm{kg})$.

\section{ACKNOWLEDGEMENTS}

This research was supported in part by the Daegu University Research Grant, 2008.

\section{REFERENCES}

Ardrey, R.E., 2003. Liquid chromatography-mass spectrometry: an introduction, pp. 98-126, Wiley, Chichester, UK.

Borges, J.H., Cabrera, J.C., Delgado, M.A.R., Suarez, E.M.H., Sauco, V.G., 2009. Analysis of pesticide residues in bananas harvested in the Canary islands (Spain), Food Chemistry 113, 313-319.

Codex Alimentarius Commission (CAC), 2003. Guidelines on good Laboratory practice in residue analysis, CAC/ GL 40-1993, Rev.1-2003, Rome, Italy.

Das, K.A., 1981. Pesticide analysis, pp. 425-453, Marcel Dekker, New York, USA.

Korea Crop Protection Association (KCPA), 2010. Agrochemicals use guide book, pp. 465-474.

Korea Food and Drug Administration (KFDA), 2009.
MRLs for pesticides in foods, p. 52.

Korea Food and Drug Administration (KFDA), 2009. Analytical methods of pesticide residues in foods - In Korean code of food, pp. 10-4-10-10-4-18 and pp. 10-4-183-10-4-184.

Lacina, O., Urbanova, J., Poustka, J., Hajaslova, J., 2010. Identification/qunatification of multiple pesticide residues in food plants by ultra-high-performance liquid chromatography-time-of-flight mass spectrometry, J. Chromatogr. A. 1217, 648-659.

Lee, Y.D., Lee, J.H., 1998. High-performance liquid chromatographic determination of tricyclazole residues in rice grain, rice straw, and soil, Agric. Chem. Biotechnol. 41, 595-599.

Lee, Y.D., Kwon, C.H., 1999. Multiresidue analysis of eight acaricides in fruits, Agric. Chem. Biotechnol. 42, 191-196.

Lee, Y.D., Kwon, C.H., 2004. Determination of monocrotophos residues in fruits and soils using high-performance liquid chromatography, Korean J. Environ. Agric. 23, 245-250.

McLafferty, F.W., Turecek, F., 1993 Interpretation of 
mass spectra, pp. 19-34, 4th ed., University Science Books, Sausalito, USA.

Pesticide Residue Analysis Research Group (PRARG), 2006. Analytical methods of pesticide residues, pp. 128-130, 2nd ed., Chuohoki Publishing, Tokyo, Japan.

Rural Development Administration (RDA), 2004. Test guidelines for pesticide persistence. In Criteria and Guidelines for Pesticide Registration, RDA Notification No. 2004-4 Annex 8, Korea.

Santos, T.F.S., Aquino, A., Dorea, H.S., Navickiene, S., 2008. MSPD procedure for determining buprofezin, tetradifon, vinclozolin, and bifenthrin residues in propolis by gas chromatography-mass spectrometry, Anal. Bioanal. Chem. 390, 1425-1430.

Snyder, L.R., Kirkland, J.J., Glajch, J.L., 1997. Practical
HPLC method development, pp. 233 266 and 292 317, 2nd ed., Wiley, New York, USA.

Soler, C., Manes, J., Pico, Y., 2005. Routine application using single quadrupole liquid chromatographymass spectrometry to pesticides analysis in citrus fruits, J. Chromatogr. A. 1088, 648-659.

Tomlin, C.D.S., 2009. The pesticide manual, pp. 138-139, 15th ed., British Crop protection Council, Hampshire, UK.

US FDA, 1999. Pesticide analytical manual, Vol 1: Multi-residue methods, 3rd ed., US Food and Drug Administration, USA.

Zweig, G., Sherma, J., 1986. Analytical methods for pesticides and plant growth regulators Vol. XV, principles, statistics, and applications, pp. 67-87, Academic Press, Orlando, USA. 